\title{
Commentary: Risk scores to predict aortic events in uncomplicated Stanford type B dissection: Simple, simpler, too simple?
}

\author{
Florian Schoenhoff, MD, and Thierry Carrel, MD
}

From the Department of Cardiovascular Surgery, University Hospital, University of Bern, Bern, Switzerland. Disclosures: Authors have nothing to disclose with regard to commercial support.

Received for publication July 10, 2019; accepted for publication July 10, 2019; available ahead of print Aug 20, 2019.

Address for reprints: Thierry Carrel, MD, Department of Cardiovascular Surgery, University Hospital University of Bern, CH-3010 Bern, Switzerland (E-mail: thierry.carrel@insel.ch).

J Thorac Cardiovasc Surg 2020;159:2184

0022-5223/\$36.00

Copyright (c) 2019 by The American Association for Thoracic Surgery

https://doi.org/10.1016/j.jtcvs.2019.07.045

Everything should be made as simple as possible, but not simpler.

-A. Einstein, 1950

Stanford type B dissection most often occurs in patients with aortic diameters that do not constitute an indication for elective surgery. When dissection has occurred, thoracic endo-vascular aortic repair (TEVAR) is the treatment of choice in patients with complicated type B dissection. The question whether patients with uncomplicated type B dissection should undergo TEVAR currently represents one of the most important gaps in evidence in our field.

The question whether to intervene in these patient has several aspects:

(1) Does TEVAR prevent future events?

(2) What is the ideal time to intervene?

(3) Are interventions for late aortic events more dangerous than the potential complications of performing TEVAR without a pressing need to do so?

In this edition of The Journal of Thoracic and Cardiovascular Surgery, Matsushita and colleagues from Chiba, Japan, try to shed light on one aspect of this complex question. The authors developed and validated a risk score that should help to identify patients at risk for late aortic events. ${ }^{1}$ The authors have shown that uncomplicated type B dissection is not a benign disease. In their patient population, the freedom from aortic events was $55 \%$ at 5 years.

The authors identified an initial aortic diameter $>40 \mathrm{~mm}$, age older than 70 years, ulcer-like projections, and larger false than true lumen diameter as the key risk factors for late aortic events. Although the authors used a "discovery" and a "validation" cohort, this was not an unbiased approach because the authors only examined a predefined set of variables.

This approach precluded the discovery of new but important risk factors for aortic events in this patient population.

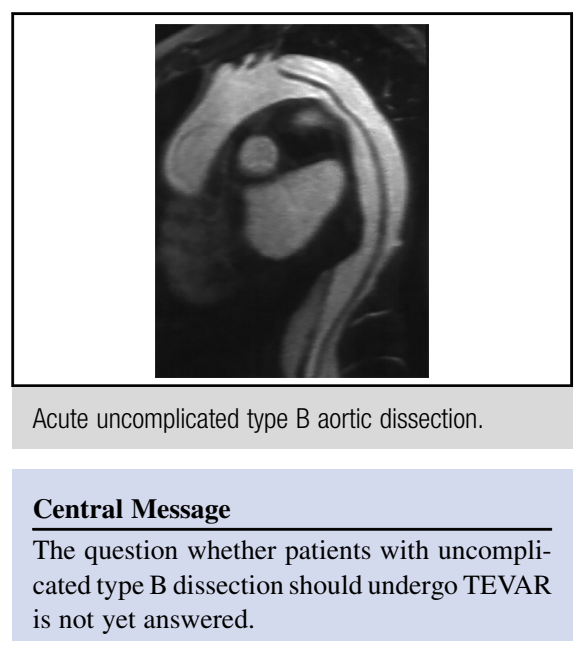

See Article page 2173 .

We know that this is an important limitation because, for example, several larger studies have identified a large entry tear $(>10 \mathrm{~mm})$ as an important risk factor for rapid dilatation; a factor that was not considered in the current study.

Risk prediction models will only be adopted into clinical practice if they have therapeutic consequences. On the basis of the data provided by the authors it is unclear whether the decision to intervene in a particular patient was on the basis the authors risk score. If the only consequence is "watchful waiting," this risk score will not help clinicians make an informed decision whether to proceed with an intervention or not. Only if the decision to intervene is on the basis of this score we will be able to judge whether late aortic events can be prevented by early intervention in patients considered to be at high risk for such events.

Although we congratulate the authors on their current study, we encourage them to initiate a randomized trial assigning patients with uncomplicated type B dissection and suitable anatomy to either best medical treatment and early intervention or best medical treatment only on the basis of their risk score. We are looking forward to the results.

\section{Reference}

1. Matsushita A, Tabata M, Mihara W, Shimamoto T, Komiya T, Takanashi S, et al. Risk score system for late aortic events in patients with uncomplicated type B aortic dissection. J Thorac Cardiovasc Surg. 2020;159:2173-83.e1. 